\title{
A double-edged sword: CRISPR-Cas9 is emerging as a revolutionary technique for genome editing
}

Chun-xiao Li and Hai-li Qian*

\begin{abstract}
In May 2015, Professor Xiao Yang authored a review on the development of CRISPR-Cas9 techniques in the journal of Military Medical Research. This review provided a valuable overview of this major scientific advance. It has been four years since the first publication of the CRISPR-Cas9 breakthrough (Science. 2012; 337: 816-21). The use of this technique has expanded into various scientific areas and is being developed into a systematic technical platform that may contribute to many bioengineering fields involving DNA sequence editing.
\end{abstract}

Keywords: CRISPR-Cas9, Genomic engineering, Ethical controversy

\section{Correspondence/findings}

Dear editor,

In May 2015, Professor Xiao Yang authored a review on the development of CRISPR-Cas9 technique in the journal of Military Medical Research [1]. This review provided a valuable overview of this major scientific advance. It has been four years since the first publication of the CRISPR-Cas9 breakthrough [2]. The use of this technique has expanded into various scientific areas and is being developed into a systematic technical platform that may contribute to many bioengineering fields involving DNA sequence editing.

The advantages of the CRISPR(Clustered Regularly Interspaced Short Palindromic Repeats)-Cas9 technology include its economy, high efficiency, precise targeting and flexible technical extension compared with traditional DNA sequence modifying measures such as transcription activator-like effector nuclease (TALEN,transcription activator-like (TAL) effector nucleases) [3]. The published literature has shown the utility of CRISPR-Cas9 in both DNA sequence knock-in and knock-out contexts. The alterations can range from single nucleotide editing to the modification of multiple genome-wide genomic sites $[4,5]$. It is easy to delete genes in cells or to create genetically modified karyotypes [6]. The CRISPR-Cas-9 strategy

\footnotetext{
* Correspondence: qianhaili001@163.com

State Key Laboratory of Molecular Oncology, Cancer Hospital, Chinese Academy of Medical Sciences, Beijing 200021, China
}

is a convenient method of screening functional genes in life processes and disease development. The technology may also be used as a potential "surgical knife" to correct genomic mutations or create new creatures by changing the inherited phenotypes. In addition to the genomic engineering applications that professor Xiao Yang mentioned, the protospacer adjacent motif (PAM) sequencelimited specificity of the CRISPR-Cas9 system has been used to circumvent engineering Cas9 derivatives. This property provides flexibility to CRISPR-Cas9 targeting strategies [7]. Poulami et al. also found another aspect of CRISPR-mediated immunity. The authors found the Type III CRISPR-Cas immune system was able to cleave DNA and RNA during infection [8]. The technique of CRISPRCas9 is still in development. If nonhomologous end joining activity is inhibited in vivo, then the efficiency of precise genome editing with CRISPR-Cas9 can be substantially increased [9].

The CRISPR-Cas9 technology does have limitations associated with targeting ability. Several off-target mutations have been detected by genome-sequencing due to its high specificity. This prohibits its potential use in correcting disease-associated mutations [10]. The rapidly expanding application of the CRISPR-Cas9 technique also creates an ethical controversy because it may be used to manipulate human germ cells. Manipulating human germ cells using CRISPR-Cas9 does not present technical obstacles. However, its potential off-target 
effects must be considered when a genetically modified individual is created with this tool. Each additional step forward will further improve the technology. Therefore, this technique should be used cautiously before modifying human inheritance. A recent publication in the journal Protein \& Cell by Junjiu Huang's group in China revealed the first attempt to modify human tripronuclear zygotes [11]. This report caused a fierce debate regarding whether this research is breaking the ethical ban on modifying the human germ cell genome [12, 13]. Simultaneously, a project led by George Church at Harvard University tried to correct genomic BRCA1(BREAST CANCER 1) mutations to decrease the risk of breast cancer in the next generation. This study was suspended indefinitely. In addition to the ethical concerns, there are technical concerns to address. The current CRISPR-Cas9 technique is not sufficiently mature to adjust human inheritance. The first issue is that CRISPR-Cas9 induces off-target changes to the genome. The second possible issue is that not all of the functions of the candidate gene are fully understood. Therefore, we cannot appreciate the consequences of genome editing in offspring.

While we are joyfully celebrating the progress brought to science by the development of CRISPR-Cas9, we must use caution in applying this technology.

\section{Competing interests}

The author declares that they have no competing interests.

\section{Authors' contributions}

CXL reviewed articles and drafted the manuscript. HLQ conceptualized the review, designed and reviewed the manuscript. Both authors read and approved the final manuscript.

\section{Authors' information}

All authors are from the State Key Laboratory of Molecular Oncology of Cancer Hospital, Chinese Academy of Medical Sciences, Beijing 100021, China. Haili Qian (PhD) is a full professor in Cancer Hospital, Chinese Academy of Medical Sciences.

\section{Acknowledgements}

This work is supported by the National Natural Science Foundation of China (81372158), National "973" Project (2015CB553904) and National "863" Project (2012AA020801). We are greatly thankful for the support.

Received: 4 June 2015 Accepted: 29 September 2015

Published online: 15 October 2015

\section{References}

1. Yang X. Applications of CRISPR-Cas9 mediated genome engineering. Mil Med Res. 2015;2:11.

2. Jinek M, Chylinski K, Fonfara I, Hauer M, Doudna JA, Charpentier E. A programmable dual-RNA-guided DNA endonuclease in adaptive bacterial immunity. Science. 2012;337:816-21.

3. Doudna JA, Charpentier E. Genome editing. The new frontier of genome engineering with CRISPR-Cas9. Science. 2014;346:1258096.

4. Ran FA, Hsu PD, Wright J, Agarwala V, Scott DA, Zhang F. Genome engineering using the CRISPR-Cas9 system. Nat Protoc. 2013:8:2281-308.

5. Shalem O, Sanjana NE, Hartenian E, Shi X, Scott DA, Mikkelsen TS, et al. Genome-scale CRISPR-Cas9 knockout screening in human cells. Science. 2014;343:84-7.
6. Guo Y, Xu Q, Canzio D, Shou J, Li J, Gorkin DU, et al. CRISPR Inversion of CTCF Sites Alters Genome Topology and Enhancer/Promoter Function. Cell. 2015;162:900-10.

7. Kleinstiver BP, Prew MS, Tsai SQ, Topkar W, Nguyen NT, Zheng Z, et al. Engineered CRISPR-Cas9 nucleases with altered PAM specificities. Nature. 2015;523:481-5.

8. Samai P, Pyenson N, Jiang W, Goldberg GW, Hatoum-Aslan A, Marraffini LA. Co-transcriptional DNA and RNA Cleavage during Type III CRISPR-Cas Immunity. Cell. 2015;161:1164-74.

9. Maruyama T, Dougan SK, Truttmann MC, Bilate AM, Ingram JR, Ploegh HL. Increasing the efficiency of precise genome editing with CRISPR-Cas9 by inhibition of nonhomologous end joining. Nat Biotechnol. 2015;33:538-42.

10. Kim D, Bae S, Park J, Kim E, Kim S, Yu HR, et al. Digenome-seq: genome-wide profiling of CRISPR-Cas9 off-target effects in human cells. Nat Methods. 2015;12:237-43.

11. Liang P, Xu Y, Zhang X, Ding C, Huang R, Zhang Z, et al. CRISPR/Cas9-mediated gene editing in human tripronuclear zygotes. Protein Cell. 2015;6:363-72.

12. Lanphier E, Urnov F, Haecker SE, Werner M, Smolenski J. Don't edit the human germ line. Nature. 2015;519:410-1.

13. Cyranoski D, Reardon S. Embryo editing sparks epic debate. Nature. 2015;520:593-4.

\section{Submit your next manuscript to BioMed Central and take full advantage of:}

- Convenient online submission

- Thorough peer review

- No space constraints or color figure charges

- Immediate publication on acceptance

- Inclusion in PubMed, CAS, Scopus and Google Scholar

- Research which is freely available for redistribution 